\title{
Health care costs and resource utilization among commercially insured adult patients with hemophilia A managed with FVIII prophylaxis in the United States
}

\author{
Courtney D Thornburg, MD, MS; Kelly Adamski, MPH; Keziah Cook, PhD; Mohini Vembusubramanian, BS; \\ Selvam R Sendhil, BA, BS; David Hinds, MHS; Er Chen, MPP; Joshua Sammon, PharmD; Paul Solari, MD; \\ Louis P Garrison, Jr, PhD; Stacy E Croteau, MD, MMS
}

\section{What is already known about this subject}

- Health care costs associated with HA are substantial and driven by the cost of FVIII concentrates.

- Cost estimates for FVIII prophylaxis have been inconsistently reported in literature. Methodology described in previous studies may incorrectly permit inclusion of patients not adhering to prophylaxis regimens.

\section{What this study adds}

- This study offers further refinement to an algorithm-based approach to identifying patients with severe $\mathrm{HA}$ within claims data.

- This study provides an updated estimate of annual treatment costs for patients with HA managed with FVIII prophylaxis using a large, commercial payer dataset. At the time of publication, estimates are the most current available and based on one of the largest cohorts of patients using administrative claims. These estimates will be critical for assessing the value of alternative treatments.

- This study highlights the substantial annual costs associated with FVIII prophylaxis as well as how different methods of identifying patients managed with FVIII prophylaxis influence the cost estimates observed.

\author{
Author affiliations \\ Courtney Thornburg, MD, MS, Division of \\ Pediatric Hematology/Oncology, Rady \\ Children's Hospital-San Diego, San Diego, \\ CA, and Department of Pediatrics, UC San \\ Diego School of Medicine, La Jolla, CA. Kelly \\ Adamski, MPH; Mohini Vembusubramanian, \\ BS; and Selvam R Sendhil, BA, BS, Analysis \\ Group, Inc., Los Angeles, CA. Keziah Cook, \\ PhD, Analysis Group, Inc., Menlo Park, CA. \\ David Hinds, MHS; Er Chen, MPP; Joshua \\ Sammon, PharmD; and Paul Solari, MD, \\ BioMarin Pharmaceutical Inc., Novato, CA. \\ Louis P Garrison, Jr, PhD, CHOICE Institute, \\ Department of Pharmacy, University of \\ Washington, Seattle. Stacy E Croteau, MD, \\ MMS, Division of Hematology-Oncology, \\ Boston Children's Hospital/Harvard Medical \\ School, Boston, MA. \\ AUTHOR CORRESPONDENCE: \\ Stacy Croteau, 617.919.2139; \\ stacy.croteau@childrens.harvard.edu
}

J Manag Care Spec Pharm. 2022;28(4):449-60

Copyright $\odot 2022$, Academy of Managed Care Pharmacy. All rights reserved. 


\section{ABSTRACT}

BACKGROUND: Standard of care for patients with severe hemophilia A (HA) is life-long prophylaxis with factor VIII (FVIII) concentrate or other hemostatic agents. Published literature highlights a wide range of treatment costs for patients with $\mathrm{HA}$.

OBJECTIVE: To estimate average annual health care costs and resource utilization for a cross-section of adult patients managed with FVIII concentrate prophylaxis using recent data from a large US commercial claims database.

METHODS: Adult males with 1 or more claim with HA diagnosis, continuous commercial plan enrollment, and 4 or more FVIII prescription dispenses during 12 months were identified from IBM MarketScan Research Database from January 2013 to September 2019, excluding those with FVIII inhibitors, an HIV/AIDS diagnosis, or diagnosis and treatment for hepatitis B or C. Patients were classified as using FVIII prophylaxis if they met any of the following definitions: (1) 6 or more FVIII dispenses, (2) a gap of 60 days or less between dispenses, and (3) at least 273 days supply in the 12 -month period. Additionally, subgroups of patients meeting each individual definition were examined, with some patients included in all 3 subgroups.

RESULTS: The overall cohort included 411 patients who met 1 or more of the 3 definitions, with a mean age of 28.9 years. Subgroups of 401,325 , and 237 patients met the first, second, and third FVIII prophylaxis definitions, respectively. Per-patient mean (SD) annual all-cause health care costs were $\$ 654,571(\$ 380,762)$ in the overall cohort and ranged from $\$ 650,065(\$ 382,196)$ to $\$ 759,661(\$ 387,040)$ among subgroups. Cost of FVIII concentrate accounted for more than $96 \%$ of total costs in the overall cohort and in each subgroup. Cost of FVIII in the overall cohort varied according to type of concentrate, with the highest among patients who were treated with both standard and extended half-life (SHL and EHL) FVIII $(\$ 784,945)$, followed by EHL FVIII only $(\$ 708,928)$, SHL FVIII only $(\$ 647,800)$, and plasmaderived FVIII $(\$ 535,614)$. The most common treatment type was SHL FVIII only (45.7\% of all patients). In the overall cohort, the majority had 1 or more outpatient visits $(94.9 \%)$, while emergency department visits, hospital admissions, and home health visits occurred less frequently $(27.0 \%, 7.1 \%$, and $7.1 \%$, respectively).

CONCLUSIONS: Commercially insured patients with HA incur substantial all-cause annual health care costs, with FVIII concentrate accounting for a majority of costs.

Hemophilia A (HA) is a congenital X-linked bleeding disorder marked by a deficiency in clotting factor VIII (FVIII) affecting more than 20,000 patients in the United States. ${ }^{1}$ FVIII activity defines the severity of the disease, ranging from mild to severe, and the aim of care is to minimize the acute and chronic complications of bleeding. For patients with severe HA, prophylaxis with FVIII concentrate or other hemostatic agents is used to prevent spontaneous bleeds, and acute bleeds are treated with additional FVIII replacement. ${ }^{2}$ The necessity of lifelong FVIII infusions poses significant cost burden, though the cost of prophylactic treatment has been inconsistently measured in the literature. The urgency to understand this cost burden has increased due to the need to assess the value of several emerging gene therapies for HA. ${ }^{3-5}$ Health economists and payers assess value in comparison to the current standard of care, therefore accurate estimates of the cost of the current standard of care are critical to that assessment. ${ }^{6-9}$

Registry and chart review studies have provided valuable insight into health care resource utilization (HRU), but the annual cost estimates are highly variable. Two studies have been conducted using national datasets from US hemophilia treatment centers. An analysis of the American Thrombosis and Hemostasis Network dataset (June 2016 through December 2017; $N=5,794$ ) estimated the annual median wholesale acquisition cost for patients with moderate and severe HA on prophylaxis to be $\$ 690,144$ or $\$ 753,480$ depending on use of standard half-life (SHL) or extended half-life (EHL) FVIII concentrate, respectively. ${ }^{10}$ This study offered a valuable benchmark for FVIII costs but assumes $100 \%$ medication adherence and uses wholesale acquisition cost pricing, potentially overestimating the annual cost of FVIII. A separate second study, which recruited pediatric and adult patients from hemophilia treatment centers (July 2005 through July 2007), reported annual average per-patient cost of \$301,392 for severe HA prophylaxis, with FVIII concentrate cost accounting for $94 \%$ of total costs. ${ }^{11}$ However, nearly half (49.5\%) of all patients in this study were aged less than 18 years, and because FVIII dosing is weight-based, the reported average costs were likely skewed lower due to the mixture of adult and pediatric patients.

Insurance claims databases represent a valuable source of cost and HRU data to assess the economic impact of hemophilia prophylaxis. Claims analyses of hemophilia have been conducted with a range of objectives, including to compare costs and utilization by inhibitor status, ${ }^{12,13}$ presence of specific symptoms, ${ }^{14,15}$ and treatment type. ${ }^{16-20}$ In these analyses, HA is generally identified by diagnosis codes International Classification of Diseases, Ninth/ Tenth Revision, Clinical Modification (ICD-9-CM: 286.0; ICD-10-CM: D66) and/or by FVIII concentrate claims. ${ }^{12,14,17,21}$ Unfortunately, identification of disease severity and inhibitor status among individuals using FVIII prophylaxis is not possible based on diagnosis codes from claims data only and instead requires algorithmic and proxy definition approaches. 
Recently, an analysis using the OptumHealth Care Solutions Claims database (January 1999 through March 2017) characterized the use of FVIII concentrates among adult patients with HA. ${ }^{22}$ That analysis reported that mean annual per-patient FVIII costs ranged from $\$ 384,000$ to $\$ 531,000$ among patients likely being managed with FVIII prophylaxis. More than $90 \%$ of overall annual health care costs were attributable to FVIII concentrate dispensed among these patients.

As a chronic, life-long condition, HA requires lifetime disease management. Current guidelines recommend prophylaxis with either FVIII or emicizumab. ${ }^{2}$ Novel treatments for HA, including gene therapy, are expected to be available for adult patients with HA in the near future. Therefore, accurate cost estimates for managing this patient population are needed to understand the economic value of new treatments.

We designed a cross-sectional analysis to estimate the annual cost of FVIII prophylaxis among adult patients to understand current treatment patterns, rather than a longitudinal study to evaluate changes over time. As the time period of the dataset was largely before the approval of emicizumab for patients with HA without inhibitors, the analysis focuses solely on FVIII prophylaxis. The goal of this analysis is to build on works published previously to provide a current and more accurate estimate of the cost of FVIII concentrate as well as overall health care costs for adult patients with HA managed with FVIII prophylaxis using a large, commercial payer dataset in the United States.

\section{Methods}

\section{DATA SOURCE}

A retrospective administrative claims analysis was conducted using the IBM MarketScan Research Database between January 2013 and September 2019. The MarketScan database includes data contributed by large employers, managed care organizations, hospitals, electronic medical record providers, Medicare, and Medicaid. The analysis focused on commercially insured patients only. The data include inpatient (IP), outpatient (OP, including emergency services), and pharmacy claims. Medical claims (IP, OP) include diagnosis codes, Current Procedural Terminology and Healthcare Common Procedure Coding System (HCPCS) codes, dates of service, place of service, and costs. Medical claims data comprise both facility and professional fees, and the information is derived from UB04 and Center for Medicare and Medicaid Services 1500 forms and their electronic equivalents, respectively. Pharmacy claims include National Drug Code (NDC) numbers, dispense dates, days supply, and costs. No institutional review board approval was necessary for the current study.

\section{SAMPLE SELECTION}

Male patients aged 18 to 64 years with HA being managed with FVIII prophylaxis who do not have evidence of active FVIII inhibitors were identified via a multistep selection process in which 3 nonmutually exclusive criteria were used to identify patients likely receiving FVIII prophylaxis during a 12-month period: (1) 6 or more FVIII dispenses of 15 or more days supply on 6 separate days, (2) a gap of 60 days or less between prescription dispenses for FVIII, (3) combined FVIII days supply of 273 or more days (sufficient FVIII for three-quarters of the year; Figure 1). These criteria were informed by previous work and refined based on initial data review by the clinical coauthors. Patients who met at least 1 of these criteria were included in the overall cohort, and subgroups of patients meeting each nonmutually exclusive criterion were examined.

Patients aged 18 to 64 years were included in the study, which comprised $65 \%$ of overall patients who met initial criteria. Younger patients were excluded over concerns that potentially less-costly FVIII could be reflective of patients weighing less, which could not be controlled for due to the lack of weight data in the dataset and would not be comparable to average adult patient costs. Medicare-eligible patients aged 65 years and older were excluded over concerns that their complete health care expenditure may not be represented in the dataset. The diagnosis of HA was identified based on ICD-9-CM/ ICD-10-CM diagnosis codes (ICD-9-CM: 286.0; ICD-10-CM: D66). A unique 12-month period of continuous enrollment for each patient was identified via an iterative process to maximize the sample size and recency of data. The most recent 12-month period of continuous enrollment with 4 or more medical or pharmacy claims for FVIII of 15 or more days supply (enough FVIII to cover prescribed dose for 15 days) in a non-IP or non-emergency department (ED) setting on 4 separate days was identified for each potentially eligible patient, with the dispense date of the first of these claims set as the index date. For example, if the most recent 12-month period had only 3 FVIII claims meeting the criteria, an earlier 12-month period was analyzed until a suitable time frame was identified, or the patient was excluded from the study. For patients who initiated prophylaxis with the nonfactor replacement treatment emicizumab (evidenced by 1 or more emicizumab dispense), the 12-month period had to occur before the first emicizumab dispense to be eligible. Patients were excluded if they had evidence of active FVIII inhibitors identified by claims for bypassing agents as patients with inhibitors often incur substantially 


\section{FIGURE 1 Sample Selection Flow Chart}

Step 1: Hemophilia diagnosis ${ }^{a}$

Patients with $\geq 1$ claim for hemophilia between Q1 2013 and Q3 2019

$\mathbf{N}=14,383$

Step 2: Nonemergent FVIII treatment

Patients with $\geq 1$ medical/pharmaceutical claim for possible prophylactic treatment in a non-IP/non-ED setting $n=3,523(24.5 \%)$

Step 3: Limit sample to male patients

Excluding all female patients $\mathrm{n}=\mathbf{2 , 9 4 6}(\mathbf{8 3 . 6 \% )}$

\section{Step 4: Age $^{\mathrm{b}}$}

Limiting to enrollment months where patients were aged between 18 and 64 years during $\geq 1$ enrollment month $n=1,901(64.5 \%)$

\section{Step 5: Continuous enrollment with sufficient FVIII treatment ${ }^{\mathrm{b}}$}

Limiting to patients from Step 4 with $\geq 4$ potential prophylactic non-IP/non-ED FVIII claims with $\geq 15$ days of supply before their earliest emicizumab claim within a period of 12 months of continuous enrollment

$$
\mathrm{n}=\mathbf{7 2 0}(37.9 \%)
$$

\section{Step 6: Excluding patients with inhibitors}

Excluding patients with evidence of bypassing agent or FVIII utilization (with $\geq 1$ drug claim for NovoSeven or Feiba)

$$
\mathrm{n}=710(98.6 \%)
$$

\section{Step 7: Excluding patients with HIV/AIDSc}

Excluding patients with any diagnosis claim for HIV/AIDS

$$
\mathrm{n}=596(83.9 \%)
$$

\section{Step 8: Excluding patients with hepatitis $\mathbf{B}$ and hepatitis $\mathbf{C}^{\mathrm{d}, \mathrm{e}}$}

Excluding patients with $\geq 1$ claim for disease and $\geq 1$ claim treatment of hepatitis $B$ or hepatitis $C$ during 12 -month period

$$
\mathrm{n}=\mathbf{5 8 3}(\mathbf{9 7 . 8 \% )}
$$

\begin{tabular}{|c|}
\hline$n=411(70.5 \%)$ \\
\hline Subgroup 2 \\
\hline $\begin{array}{l}\text { Patients whose maximum gap } \\
\text { in days supply } \leq 60 \text { days } \\
\text { in } 12 \text {-month analytic period }\end{array}$ \\
\hline$n=325(79.1 \%)$ \\
\hline
\end{tabular}

\section{Step 9: Patients managed with FVIII prophylaxis (overall cohort)}

\begin{tabular}{|c|}
\hline Subgroup 3 \\
\hline $\begin{array}{c}\text { Patients with supply of FVIII lasting } \\
\text { at least three-quarters of their } \\
\text { 12-month analytic period }\end{array}$ \\
\hline$n=237(57.7 \%)$ \\
\hline
\end{tabular}

Limiting to patients that meet the inclusion criteria for $\geq 1$ of the 3 subgroups of patients likely receiving FVIII prophylaxis

Subgroup $\mathbf{1}$
Patients with $\geq 6$ distinct
non-IP/non-ED FVIII claims in
12-month analytic period
$\mathbf{n}=\mathbf{4 0 1}(\mathbf{9 7 . 6 \% )}$

aClaims for hemophilia were identified with the following diagnosis codes: ICD-9-CM: 286.0; ICD-10-CM: D66.

${ }^{b}$ Patients had at least 4 potential prophylactic non-IP/non-ED FVIII claims with more than 15 days supply each on at least 4 different dates.

'Claims for HIV/AIDS were identified with the following diagnosis codes: ICD-9-CM: 042-044; ICD-10-CM: B20-B22, B24.

${ }^{d}$ Claims for hepatitis B were identified with the following diagnosis codes: ICD-9-CM: 070.2, 070.30-33; ICD-10-CM: B19.1, B18.0-1.

${ }^{e}$ Claims for hepatitis $C$ were identified with the following diagnosis codes: ICD-9-CM: 070.41, 070.44, 070.51, 070.54, 070.7; ICD-10-CM: B17, B18.2, B19.2.

$E D=$ emergency department; FVII = factor VII; FVIII = factor VIII; ICD-9/10-CM=International Classification of Diseases, Ninth/Tenth, Clinical Modification;

$I P=$ inpatient; $Q=$ quarter. 


\section{TABLE 1 Patient Characteristics}

\begin{tabular}{|c|c|c|c|c|c|c|c|c|}
\hline \multirow{2}{*}{$\begin{array}{ll}\text { Characteristic } \\
\text { Age, mean (SD), y }\end{array}$} & \multicolumn{2}{|c|}{$\begin{array}{l}\text { Overall cohort } \\
\qquad(\mathrm{N}=411)^{\mathrm{a}}\end{array}$} & \multicolumn{2}{|c|}{$\begin{array}{l}\text { Subgroup } 1 \\
\geq 6 \text { FVIII dispenses } \\
(n=401)\end{array}$} & \multicolumn{2}{|c|}{$\begin{array}{l}\text { Subgroup } 2 \\
\leq 60 \text {-day gap } \\
(n=325)\end{array}$} & \multicolumn{2}{|c|}{$\begin{array}{l}\text { Subgroup } 3 \\
\geq 273 \text { days of supply } \\
(n=237)\end{array}$} \\
\hline & 28.9 & (8.4) & 28.9 & $(8.4)$ & 28.9 & $(8.2)$ & 29.3 & $(8.4)$ \\
\hline $18-25, \mathrm{n}(\%)$ & 198 & $(48.2)$ & 192 & $(47.9)$ & 151 & $(46.5)$ & 106 & $(44.7)$ \\
\hline $26-64, \mathrm{n}(\%)$ & 213 & $(51.8)$ & 209 & $(52.1)$ & 174 & $(53.5)$ & 131 & $(55.3)$ \\
\hline \multicolumn{9}{|l|}{ Year at beginning of study period, $n(\%)$} \\
\hline 2013 & 55 & $(13.4)$ & 54 & $(13.5)$ & 46 & $(14.2)$ & 34 & $(14.3)$ \\
\hline 2014 & 43 & $(10.5)$ & 40 & $(10.0)$ & 31 & $(9.5)$ & 24 & $(10.1)$ \\
\hline 2015 & 49 & $(11.9)$ & 47 & $(11.7)$ & 35 & $(10.8)$ & 26 & $(11.0)$ \\
\hline 2016 & 52 & $(12.7)$ & 51 & $(12.7)$ & 41 & $(12.6)$ & 36 & $(15.2)$ \\
\hline 2017 & 53 & $(12.9)$ & 51 & $(12.7)$ & 42 & $(12.9)$ & 33 & $(13.9)$ \\
\hline 2018 & 159 & $(38.7)$ & 158 & $(39.4)$ & 130 & $(40.0)$ & 84 & $(35.4)$ \\
\hline \multicolumn{9}{|l|}{ Beneficiary status, n (\%) } \\
\hline Dependent & 220 & $(53.5)$ & 211 & $(52.6)$ & 170 & $(52.3)$ & 122 & $(51.5)$ \\
\hline Employee & 191 & $(46.5)$ & 190 & $(47.4)$ & 155 & $(47.7)$ & 115 & $(48.5)$ \\
\hline \multicolumn{9}{|l|}{ Region, n (\%) } \\
\hline North Central & 96 & $(23.4)$ & 91 & $(22.7)$ & 75 & $(23.1)$ & 54 & $(22.8)$ \\
\hline Northeast & 70 & $(17.0)$ & 67 & $(16.7)$ & 53 & $(16.3)$ & 37 & $(15.6)$ \\
\hline South & 173 & $(42.1)$ & 172 & $(42.9)$ & 137 & $(42.2)$ & 96 & $(40.5)$ \\
\hline West & 69 & $(16.8)$ & 68 & $(17.0)$ & 58 & $(17.8)$ & 49 & $(20.7)$ \\
\hline Unknown & 3 & $(0.7)$ & 3 & $(0.7)$ & 2 & $(0.6)$ & 1 & $(0.4)$ \\
\hline \multicolumn{9}{|c|}{ Insurance type at beginning of study period, $n(\%)$} \\
\hline Capitated or partially capitated POS plan & 7 & $(1.7)$ & 7 & $(1.7)$ & 6 & $(1.8)$ & 5 & $(2.1)$ \\
\hline CDHP & 31 & $(7.5)$ & 30 & $(7.5)$ & 26 & $(8.0)$ & 25 & $(10.5)$ \\
\hline Comprehensive plan & 6 & $(1.5)$ & 6 & $(1.5)$ & 5 & $(1.5)$ & 4 & $(1.7)$ \\
\hline EPO plan & 2 & $(0.5)$ & 2 & $(0.5)$ & 2 & $(0.6)$ & 1 & $(0.4)$ \\
\hline HDHP & 43 & $(10.5)$ & 42 & $(10.5)$ & 32 & $(9.8)$ & 25 & $(10.5)$ \\
\hline HMO plan & 38 & $(9.2)$ & 38 & $(9.5)$ & 32 & $(9.8)$ & 20 & $(8.4)$ \\
\hline Non-capitated POS plan & 31 & $(7.5)$ & 30 & $(7.5)$ & 27 & $(8.3)$ & 22 & $(9.3)$ \\
\hline PPO plan & 241 & $(58.6)$ & 234 & $(58.4)$ & 188 & $(57.8)$ & 130 & $(54.9)$ \\
\hline Unknown & 12 & $(2.9)$ & 12 & (3.0) & 7 & $(2.2)$ & 5 & $(2.1)$ \\
\hline
\end{tabular}

higher cost than patients without inhibitors. Patients with a diagnosis of HIV/AIDS or those with hepatitis B (HBV) or hepatitis $\mathrm{C}(\mathrm{HCV})$ receiving hepatitis treatment $(\geq 1$ claim for hepatitis treatment during the 12-month period) were also excluded. These patients may require additional care to manage their comorbid condition. A complete list of included ICD-9-CM/ICD-10-CM diagnosis codes; FVIII products (NDC, HCPCS), excluded bypassing agents (NDC); and excluded HBV/HCV treatment (NDC) are provided in Supplementary Tables 1-6 (available in online article).

After applying the above selection criteria, patients were evaluated under 3 prophylaxis subgroup criteria. The first subgroup was based on quantity of claims, requiring that patients have 6 or more non-IP, non-ED FVIII claims with 15 or more days supply. The second subgroup was based on frequency of claims, requiring no supply gaps more than 
TABLE 1 Patient Characteristics (continued)

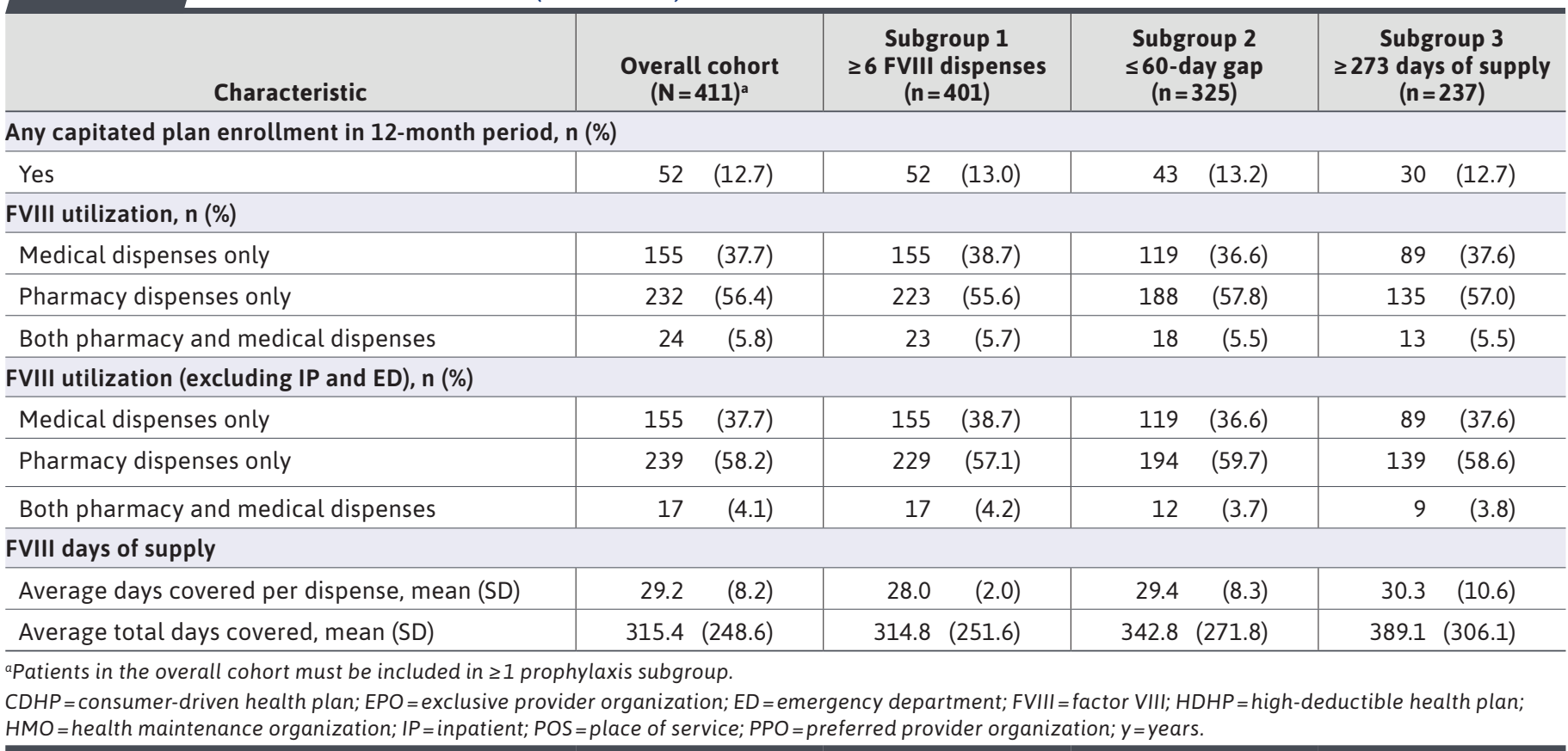

60 days between fills, after accounting for days supply. The third subgroup was based on days of coverage, requiring patients have sufficient FVIII for at least three-fourths of the year.

Dispenses for the same FVIII product on the same day were assumed to represent a combination of vial sizes for patients to achieve their necessary dose. Therefore, the maximum days supply for the 2 products was assumed to be that date's days supply. The mode of days supply by product calculated from pharmacy claims was used to impute the days supply for product-specific HCPCS codes identified in the medical claims. HCPCS codes for products without corresponding pharmacy claims were assumed to have 28 days supply as, typically, prophylaxis prescriptions are written to dispense 28 days supply. Total doses of supply may vary based on the actual treatment regimen.

The patient selection algorithm was informed by previously published work analyzing OptumHealth Care Solutions claims and was further modified to refine the identification of patients prescribed FVIII prophylaxis therapy. ${ }^{22}$ Analysis of previous datasets did not include an iterative effort to identify the most recent 12-month period that met the criteria number of FVIII fills. The larger sample resulting from the use of IBM MarketScan database and an iterative process of identifying patients allowed us to test
3 operational definitions for FVIII prophylaxis, including 1 based on days of coverage.

\section{STUDY OUTCOMES}

Health care cost and HRU outcomes for the 12-month analytic period were reported for the overall cohort and for each of the 3 subgroups. Health care cost outcomes included all-cause total medical and pharmacy costs as well as total FVIII concentrate claims (including either medical or pharmacy claims). Health care resource utilization outcomes included frequency of claims by place of service, specifically IP, ED, OP, and home health. All costs were reported in 2020 US dollars (USD) and adjusted for inflation using the medical care component of the US Consumer Price Index. ${ }^{23}$

\section{STATISTICAL ANALYSES}

Descriptive statistics were computed for patient characteristics and each health care cost and HRU outcome. Patient characteristics include age, insurance plan type, beneficiary status, time with insurance coverage, and region of insurance coverage. A sensitivity analysis of cost outcomes was conducted that excluded patients enrolled in a capitated plan at some point during their eligibility period, because patients enrolled in capitated plans are not expected to have their full medical costs represented in the data. 


\section{FIGURE 2 Average Annual Per-Patient Health Care Costs}

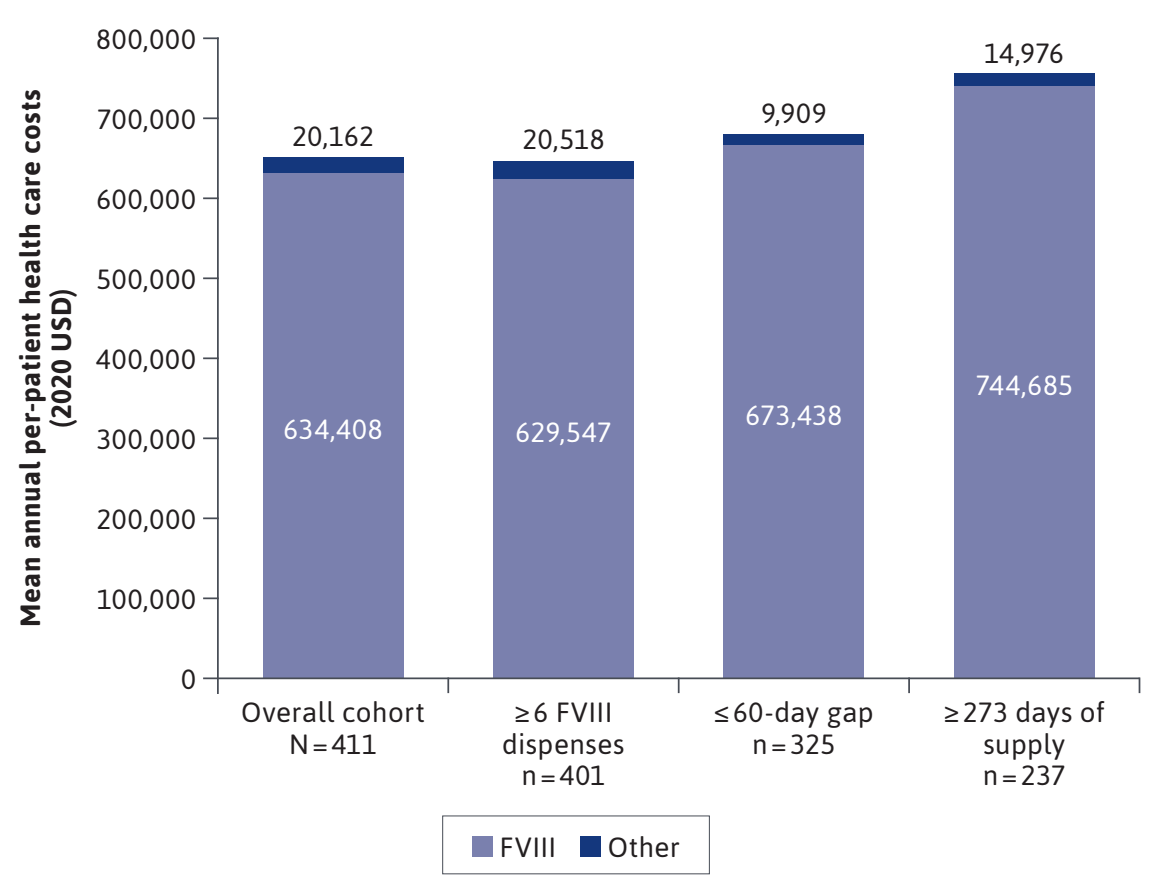

FVIII = factor VIII; USD = US dollar

\section{Results}

\section{PATIENT CHARACTERISTICS}

A total of 1,901 adult male patients with a diagnosis of $\mathrm{HA}$ and at least 1 claim for FVIII concentrate were identified from the database from January 2013 through September 2019 (Figure 1). The overall cohort after applying exclusion criteria included 411 adult male patients with HA managed with FVIII prophylaxis. Among the overall cohort, a total of 401 patients had 6 or more FVIII dispenses during the 12-month eligibility period (subgroup 1), 325 patients had no supply gaps more than 60 days between FVIII dispenses during the 12-month eligibility period (subgroup 2), and 237 patients had a supply of FVIII lasting at least three-quarters of the 12-month eligibility period (subgroup 3). All patients in the overall cohort met 1 or more of the criteria, and 222 patients met all 3 criteria.

The mean (SD) age among the overall cohort was 28.9 (8.4) years (Table 1). Patients in the overall cohort were most commonly insured by a preferred provider organization plan (58.6\%), followed by high-deductible health plan (10.5\%), health maintenance organization plan (9.2\%), consumer-driven health plan $(7.5 \%)$, and noncapitated point-of-service plan (7.5\%). More patients in the overall cohort had FVIII (excluding FVIII administered in IP and ED settings) dispensed through pharmacy benefits (58.2\%) than medical benefits $(37.7 \%)$, and few patients had both pharmacy and medical dispenses (4.1\%). In the overall cohort, annual mean (SD) total days covered by FVIII was 315.4 (248.6) days, with a mean (SD) days covered per dispense of 29.2 (8.2) days.

\section{HEALTH CARE COSTS}

Cost of FVIII concentrate was the primary cost driver, accounting for more than $96 \%$ of total health care costs in the overall cohort and across the 3 prophylaxis subgroups with alternate criteria for identifying patients being managed with FVIII prophylaxis (Figure 2). Per-patient mean (SD) total annual all-cause health care costs for the overall cohort were $\$ 654,571$ $(\$ 380,762)$, and $\$ 650,065 \quad(\$ 382,196)$ for subgroup 1, $\$ 683,347(\$ 366,171)$ for subgroup 2, and $\$ 759,661(\$ 387,040)$ for subgroup 3 (Table 2). Per-patient mean (SD) annual FVIII cost was $\$ 634,408$ $(\$ 358,566)$ in the overall cohort, and $\$ 629,547 \quad(\$ 359,394), \quad \$ 673,438$ $(\$ 365,531)$, and $\$ 744,685 \quad(\$ 378,601)$ among patients meeting the respective criteria for prophylaxis. Costs of FVIII were similar when excluding treatment occurring in IP and ED settings with per-patient mean (SD) annual FVIII cost of $\$ 633,387(358,249)$ in the overall cohort, and $\$ 628,519$ (\$359,079), \$672,372 (\$365,438), and $\$ 743,284$ (\$378,446) among patients meeting the respective criteria for prophylaxis.

More patients were dispensed FVIII (non-IP/non-ED) through pharmacy benefits than medical benefits in the overall cohort (58.2\% vs $37.7 \%$ ), and these patients had higher mean (SD) non-IP/non-ED FVIII costs (\$653,099 $[\$ 351,886]$ vs $\$ 541,446 \quad[\$ 384,118]$, respectively). Similar trends were observed across the 3 prophylaxis subgroups as well. Additional health care costs, including the median (IQR) for all presented categories, are provided in Supplementary Table 7.

Average annual FVIII cost varied according to type of FVIII concentrate. Among the overall cohort, average annual per-patient FVIII costs were highest among patients who 


\begin{tabular}{|c|c|c|c|c|}
\hline $\begin{array}{c}\text { Health care } \\
\text { cost } \\
\text { category }\end{array}$ & $\begin{array}{l}\text { Overall cohort } \\
\qquad(\mathrm{N}=411)\end{array}$ & $\begin{array}{l}\text { Subgroup } 1 \\
\geq 6 \text { FVIII dispenses } \\
(n=401)\end{array}$ & $\begin{array}{c}\text { Subgroup } 2 \\
\leq 60 \text {-day gap } \\
(n=325)\end{array}$ & $\begin{array}{c}\text { Subgroup } 3 \\
\geq 273 \text { days of supply } \\
(n=237)\end{array}$ \\
\hline \multicolumn{5}{|c|}{ Il-cause total health care cost $\mathrm{t}^{\mathrm{b}}$} \\
\hline Mean (SD) & $654,571(380,762)$ & $650,065(382,196)$ & $683,347(366,171)$ & $759,661(387,040)$ \\
\hline \multicolumn{5}{|c|}{ VIII cost (pharmacy and medical) } \\
\hline (SD) & $634,408(358,566)$ & $629,547(359,394)$ & $673,438(365,531)$ & $744,685(378,601)$ \\
\hline
\end{tabular}

FVIII cost (pharmacy only)

\begin{tabular}{l|c|c|c|c}
\hline $\mathrm{n}$ & 256 & 246 & 206 & 148 \\
\hline Mean (SD) & $653,099(351,886)$ & $645,965(353,409)$ & $687,027(356,947)$ & $759,702(370,982)$ \\
\hline
\end{tabular}

FVIII cost (medical only)

\begin{tabular}{l|c|c|c|c}
\hline $\mathrm{n}$ & 179 & 178 & 137 & 102 \\
\hline Mean (SD) & $522,616(390,280)$ & $525,511(389,450)$ & $564,523(406,950)$ & $627,985(427,897)$ \\
\hline
\end{tabular}

FVIII cost (pharmacy and medical, excluding IP and ED)

\begin{tabular}{|l|l|l|l|l} 
Mean (SD) & $633,387(358,249)$ & $628,519(359,079)$ & $672,372(365,438)$ & $743,284(378,446)$ \\
\hline
\end{tabular}

FVIII cost (pharmacy only, excluding IP and ED) ${ }^{c}$

\begin{tabular}{l|c|c|c|c}
\hline $\mathrm{n}$ & 256 & 246 & 206 & 148 \\
\hline Mean (SD) & $653,099(351,886)$ & $645,965(353,409)$ & $687,027(356,947)$ & $759,702(370,982)$ \\
\hline
\end{tabular}

FVIII cost (medical only, excluding IP and ED)c

\begin{tabular}{l|c|c|c|c}
\hline $\mathrm{n}$ & 172 & 172 & 131 & 98 \\
\hline Mean (SD) & $541,446(384,118)$ & $541,446(384,118)$ & $587,736(399,232)$ & $650,229(418,413)$ \\
\hline
\end{tabular}

Note: Costs are in 2020 USD.

apatients in the overall cohort must be included in $\geq 1$ prophylaxis subgroup.

${ }^{b}$ All-cause total health care costs are the sum of the mutually exclusive categories of total FVIII costs, total medical costs (excluding medical FVIII), and non-FVIII pharmacy costs.

'FVIII pharmacy costs and FVIII medical costs are calculated only among patients with $\geq 1$ FVIII pharmacy or medical claim, respectively.

$E D=$ emergency department; FVIII = factor VIII; IP = inpatient; USD = United States dollars.

treated with both SHL and EHL FVIII (\$784,945), followed by EHL FVIII only (\$708,928), SHL FVIII only (\$647,800), and plasma-derived FVIII $(\$ 535,614)$. Patients were more likely to treat with SHL FVIII only (45.7\%), followed by EHL FVIII only (20.7\%), SHL and EHL FVIII (3.6\%), and plasma-derived FVIII (2.2\%). In roughly $28 \%$ of patients, the type of FVIII concentrate was billed under a HCPCS code that does not identify the specific product, and these patients had an average annual per-patient FVIII cost of $\$ 544,753$. When patients with nonspecific types of FVIII concentrate usage were excluded, proportion of patients treated with SHL FVIII only, EHL FVIII only, both SHL and EHL FVIII, and plasma-derived FVIII were $63.3 \%$, $28.6 \%, 5.1 \%$, and $3.0 \%$, respectively. A summary of per-patient annual FVIII concentrate usage is provided in Supplementary Table 8. A complete list of included FVIII products is provided in Supplementary Tables 2 and 3.

A sensitivity analysis of cost outcomes that excluded patients enrolled in a capitated plan at some point during their eligibility period had minimal effect on annual per-patient cost estimates.

\section{HEALTH CARE RESOURCE UTILIZATION}

Aside from FVIII concentrate, the most common all-cause HRU were OP visits, followed by ED visits, IP admissions, and home health visits (Table 3 ). The majority of patients in the overall cohort had 1 or more OP visit (94.9\%), whereas patients utilized ED visits, IP admissions, and home health visits less frequently $(27.0 \%, 7.1 \%$, and $7.1 \%$, respectively). Annually, patients with 1 or more utilization had a mean (SD) of 17.4 (23.8) home health visits, 9.0 (11.8) OP visits, 1.9 (1.8) ED visits, and 1.3 (0.9) IP admissions. In the overall cohort, $62.3 \%$ of patients had 1 or more pharmacy claim and $41.8 \%$ had 1 or more medical claim for FVIII concentrate (excluding FVIII administered in IP and ED settings). Annual mean (SD) number of FVIII (non IP/non-ED) dispenses was 11.1 (8.9) among the overall cohort. Trends in HRU were generally similar across prophylaxis definition subgroups.

\section{Discussion}

The current study furthers published work and provides updated cost and HRU estimates for US patients with HA managed with FVIII prophylaxis using a large, commercial payer dataset. Of importance, the study illustrates the significance of identifying patients who are likely on FVIII prophylaxis when using claims data. Health care costs associated with HA are substantial, varying from a per-patient annual cost of $\$ 650,065$ to $\$ 759,661$ depending on the criteria used to identify FVIII prophylaxis use. The cost of FVIII concentrate accounts for more than $96 \%$ of total health care costs among patients in the overall cohort and across the 3 prophylaxis subgroups with alternate criteria for identifying patients being managed with FVIII prophylaxis. 
TABLE 3 Health Care Resource Utilization

\begin{tabular}{|c|c|c|c|c|}
\hline $\begin{array}{c}\text { All-cause } \\
\text { HRU category }\end{array}$ & $\begin{array}{l}\text { Overall cohort } \\
(\mathrm{N}=411)\end{array}$ & $\begin{array}{c}\text { Subgroup } 1 \\
\geq 6 \text { FVIII } \\
\text { dispenses } \\
(n=401)\end{array}$ & $\begin{array}{c}\text { Subgroup } 2 \\
\leq 60 \text {-day } \\
\text { gap } \\
(n=325)\end{array}$ & $\begin{array}{l}\text { Subgroup } 3 \\
\geq 273 \text { days } \\
\text { of supply } \\
\text { ( } n=237)\end{array}$ \\
\hline \multicolumn{5}{|c|}{ Number of IP admissions ${ }^{b}$} \\
\hline Patients, n (\%) & $29(7.1)$ & $29(7.2)$ & $21(6.5)$ & $17(7.2)$ \\
\hline Mean (SD) & $1.3(0.9)$ & $1.3(0.9)$ & $1.0(0.0)$ & $1.2(1.0)$ \\
\hline Median (IQR) & $1.0(1.0-1.0)$ & $1.0(1.0-1.0)$ & $1.0(1.0-1.0)$ & $1.0(1.0-1.0)$ \\
\hline \multicolumn{5}{|l|}{ Number of ED visits ${ }^{b}$} \\
\hline Patients, n (\%) & $111(27.0)$ & $110(27.4)$ & $92(28.3)$ & $60(25.3)$ \\
\hline Mean (SD) & $1.9(1.8)$ & $1.9(1.8)$ & $1.7(1.2)$ & $1.7(1.1)$ \\
\hline Median (IQR) & $1.0(1.0-2.0)$ & $1.0(1.0-2.0)$ & $1.0(1.0-2.0)$ & $1.0(1.0-2.0)$ \\
\hline \multicolumn{5}{|l|}{ Number of OP visits ${ }^{b}$} \\
\hline Patients, n (\%) & $390(94.9)$ & $382(95.3)$ & $308(94.8)$ & $222(93.7)$ \\
\hline Mean (SD) & $9.0(11.8)$ & $9.0(11.8)$ & $9.0(12.2)$ & $9.9(14.1)$ \\
\hline Median (IQR) & $5.0(2.0-11.0)$ & $5.0(2.0-11.0)$ & $5.0(2.0-10.0)$ & $5.0(2.0-11.0)$ \\
\hline \multicolumn{5}{|c|}{ Number of home health visits } \\
\hline Patients, n (\%) & $29(7.1)$ & $29(7.2)$ & $22(6.8)$ & $15(6.3)$ \\
\hline Mean (SD) & $17.4(23.8)$ & $17.4(23.8)$ & $20.5(26.6)$ & $21.4(26.3)$ \\
\hline Median (IQR) & $9.0(7.0-11.0)$ & $9.0(7.0-11.0)$ & $10.0(8.0-11.0)$ & $10.0(9.5-11.5)$ \\
\hline \multicolumn{5}{|c|}{ Number of pharmacy FVIII claims ${ }^{c}$} \\
\hline Patients, n (\%) & $256(62.3)$ & $246(61.3)$ & $206(63.4)$ & $148(62.4)$ \\
\hline Mean (SD) & $9.9(3.0)$ & $10.1(2.8)$ & $10.5(2.8)$ & $11.4(2.8)$ \\
\hline Median (IQR) & $10.0(8.0-12.0)$ & $10.0(8.0-12.0)$ & $11.0(9.0-12.8)$ & $12.0(11.0-13.0)$ \\
\hline \multicolumn{5}{|c|}{ Number of medical FVIII claims ${ }^{c}$} \\
\hline Patients, n (\%) & $172(41.8)$ & $172(42.9)$ & $131(40.3)$ & $98(41.4)$ \\
\hline Mean (SD) & $11.7(13.6)$ & $11.7(13.6)$ & $13.2(15.2)$ & $15.3(17.1)$ \\
\hline Median (IQR) & $10.0(7.0-12.0)$ & $10.0(7.0-12.0)$ & $11.0(9.0-13.0)$ & $12.0(11.0-14.0)$ \\
\hline \multicolumn{5}{|c|}{$\begin{array}{l}\text { aPatients in the overall cohort must be included in } \geq 1 \text { prophylaxis subgroup. } \\
\text { 'Only patients with } \geq 1 \text { utilization in each category were included in descriptive statistics (ie, mean, } \\
S D, I Q R \text { ). } \\
\text { 'Excludes IP and ED FVIII claims. } \\
E D=\text { emergency department; FVIII = factor VIII; HRU = health care resource utilization; IP=inpatient; } \\
I Q R=\text { interquartile range; OP=outpatient. }\end{array}$} \\
\hline
\end{tabular}

Cost estimates from the current study are likely to be the best estimates of commercial insurer paid costs currently available. Annual costs estimates for FVIII prophylaxis were previously reported in a study using MarketScan data. The 2017 study reported hemophilia-related mean total annual health care costs among patients aged 19-44 years to be $\$ 256,426$ for those managed with prophylaxis and $\$ 197,732$ for their nonprophylaxis counterparts..$^{14}$ However, the study excluded all patients with combined annual days supply more than 157 days from the prophylaxis subgroup, essentially removing from their sample all patients adhering to prophylaxis regimens described in clinical guidelines.
More recently, an analysis using the OptumHealth Care Solutions Claims database characterized the use of FVIII concentrates among adult patients with HA. ${ }^{22}$ The analysis reported that the mean annual per-patient FVIII costs ranged from $\$ 384,000$ to $\$ 531,000$, among patients likely being managed with FVIII prophylaxis and after accounting for proxies for inhibitor status. FVIII claims account for more than $90 \%$ of overall health care costs among these patients. However, the analysis was conducted on a smaller dataset over an earlier timeframe (1999-2017) and included a less restrictive definition of prophylaxis ( $\geq 4$ FVIII dispenses per year), which likely captured some patients receiving only episodic FVIII therapy. FVIII can be administered both as episodic treatment and prophylactic treatment. Although 4 FVIII claims with 90 days supply would be the minimum needed for prophylactic usage in a single year, increasing the lower threshold to 6 FVIII claims improves the likelihood of only capturing patients on prophylaxis in the sample.

In the current analysis, mean (SD) cost estimates of non-IP/non-ED FVIII were higher for FVIII dispensed through pharmacy benefits compared with medical benefits in the overall cohort $(\$ 653,099[\$ 351,886]$ vs $\$ 541,446[\$ 384,118])$, suggesting medical FVIII costs in the MarketScan data may reflect net costs after accounting for discounts and rebates whereas pharmacy FVIII costs in these data may not.

Analysis of per-patient annual FVIII cost by concentrate type showed that costs were highest among patients who treated with both SHL and EHL FVIII $(\$ 784,945)$, followed by EHL FVIII only (\$708,928), SHL FVIII only $(\$ 647,800)$, and plasma-derived FVIII $(\$ 535,614)$. Patients more likely treated 
with SHL FVIII only (63.3\%), followed by EHL FVIII only (28.6\%), SHL and EHL FVIII (5.1\%), and plasma-derived FVIII (3.0\%), when excluding patients with nonspecific types of FVIII concentrate usage. Increased utilization and higher costs of EHL FVIII may account for some of the differences compared with estimates based on older data in the literature.

A sensitivity analysis was conducted to exclude patients enrolled in a capitated plan at some point during their eligibility period from the overall cohort. However, the exclusion of patients enrolled in a capitated plan from the analysis had minimal effect on health care cost outcomes, likely because cost of FVIII is being adequately captured and FVIII cost accounts for the majority of health care costs.

As noted above, the emergence of several gene therapies for HA is raising questions about their potential "value for money" (or cost-effectiveness, in health economic terminology). These new treatments, if approved, would be an alternative to prophylaxis with FVIII concentrate or nonfactor therapies and could reduce utilization and associated treatment cost while maintaining adequate protective FVIII activity level. One analysis of a hemophilia gene therapy projected a median durability of 10.7 years before resuming FVIII prophylaxis and that therapy would be cost-saving (by $\$ 6.8$ million over a lifetime). ${ }^{7}$ Modeling the same gene therapy, the Institute for Clinical and Economic Review (ICER) also projected that the gene therapy would be costsaving (by $\$ 5$ million over a lifetime) and produce greater health gain compared with FVIII prophylaxis. ${ }^{9}$ In the ICER analysis, the mean annual drug cost of FVIII prophylaxis was modeled to be approximately $\$ 630,000$. Our real-world claims analysis suggests that this may be an underestimate of annual FVIII prophylaxis cost depending on the measurement definition-with over one-half of the sample averaging annual costs of nearly $\$ 750,000$. In any case, an accurate assessment of the potential value of any prophylactic therapy will critically depend on reliable estimates of the cost of the standard of care, while recognizing the methodologic challenges and inherent uncertainties in these needed forecasts.

\section{LIMITATIONS}

Several limitations should be considered when interpreting the results of this analysis. The cross-sectional design of this study did not provide a direct estimate of the longitudinal or lifetime costs of FVIII treatment. However, given that HA is a chronic disease and that patients are on longterm treatment, and, furthermore, that the included sample included a wide range of ages, the mean annual cost would likely provide the basis for a reasonable approximation of the longer-term costs of FVIII treatment in these adults.

Administrative claims data lacked details associated with clinical or laboratory data that would have provided information on timing of actual administration of FVIII relative to time of dispensing, the prescribed dose, use of FVIII for continuous or intermittent prophylaxis, documented use of prophylaxis, clinical and laboratory confirmation of disease severity and presence of inhibitors, and indication of home health visits. Therefore, there was a trade-off between the potential misclassification of patients with HA managed with FVIII prophylaxis and more restrictive identification criteria. The ICD-9-CM code for HA is nonspecific for HA and captures patients with other bleeding disorders as well. During sample selection, patients with HA were inferred by limiting to those with at least 1 medical or pharmacy claim for FVIII along with other factors consistent with previously published work. ${ }^{21}$ Patients managed with prophylactic FVIII may have been inadvertently excluded from the overall cohort because their FVIII dispensing pattern was not evenly spaced enough to be captured by 1 of the 3 definitions for FVIII prophylaxis.

Patients with inhibitors may also have been present in the study sample despite having defined criteria for their exclusion. During sample selection, positive inhibitor status was inferred based on a claims record for bypassing agents. Patients who had achieved partial tolerance and used high doses of FVIII without bypassing agents may have been incorrectly included.

The reported costs do not reflect patients managed with emicizumab prophylaxis. For patients in the overall cohort who initiated prophylaxis with the nonfactor replacement treatment emicizumab, only FVIII dispenses that occurred before the first emicizumab dispense were assessed for eligibility as the study time period did not sufficiently capture emicizumab's approval for prophylaxis in the noninhibitor HA population. Reported costs also do not reflect patients with known comorbidities, specifically HIV and HBV/HCV. However, we would not anticipate that FVIII-specific costs would differ for these patients.

Patients who were dispensed a prophylaxis dose and a pro re nata dose for the same FVIII product on the same day were assumed to represent a combination of vial sizes for patients to achieve their necessary dose.

The analysis was limited to males with HA, although females comprised a small proportion of the HA population. Given that FVIII dosing is weight-based, annual cost of FVIII is likely to be lower for females with HA as they are generally lighter than males. 
Finally, the reported costs reflect commercial beneficiaries and may not be representative of beneficiaries enrolled in public health plans such as Medicaid or the military health plan Tricare. Analysis of claims databases that contain such data could facilitate refinement to the described algorithm and associated criteria for identifying patients likely to be managed with FVIII prophylaxis.

\section{Conclusions}

The current study provides updated cost and HRU estimates for patients with HA managed with FVIII prophylaxis. The mean per-patient total annual all-cause health care cost for the overall cohort was $\$ 654,571$ and ranged from $\$ 650,065$ to $\$ 759,661$ depending on the definition of prophylaxis. Consistent with previous literature, cost of FVIII concentrate is a primary cost driver, accounting for more than $96 \%$ of overall health care costs in the overall cohort and across the 3 prophylaxis subgroups defined according to the different criteria for prophylaxis usage. Type of FVIII product also influenced costs. The study additionally highlights the impact of how definitions used will influence the estimates observed, particularly for conditions like HA. Finally, the study provides a reliable benchmark for health insurers to monitor their expenditures and allows a basis for assessing the clinical and economic value of new and emerging technologies that aim to improve the HA treatment paradigm. ${ }^{9,24-26}$

\section{DISCLOSURES}

This study was funded by BioMarin Pharmaceutical Inc, which was involved in the protocol development, analysis plan development, data interpretation, manuscript preparation, and publication decisions. All authors contributed to protocol development, analysis plan development, data interpretation, and manuscript development and maintained control over the final content.

Thornburg has received professional fees from BioMarin Pharmaceutical, CSL Behring, Genentech, Novo Nordisk, Sanofi Genzyme, HEMA Biologics, and Spark Therapeutics and institutional research funding from BioMarin Pharmaceutical, Novo Nordisk, and Sanofi Genzyme. Adamski, Cook, and Sendhil are employees of Analysis Group, a consulting company that was contracted by BioMarin Pharmaceutical to conduct this study and develop the manuscript. Vembusubramanian is a former employee of Analysis Group. Hinds, Chen, and Sammon are employees and shareholders of BioMarin Pharmaceutical. Solari is a former employee of BioMarin Pharmaceutical. Garrison has received consulting fees from BioMarin Pharmaceutical and Analysis Group. Croteau has received professional fees from BioMarin Pharmaceutical, Bayer, CSL Behring, HEMA Biologics, and Pfizer Inc and institutional research funding from Novo Nordisk and Spark Therapeutics.

\section{ACKNOWLEDGMENTS}

The authors thank Deepshekhar Gupta, an employee at Analysis Group, for helping with the data analysis, and Taiji Wang, an employee at Analysis Group, for providing medical writing assistance.

\section{REFERENCES}

1. Centers for Disease Control and Prevention. Community counts: factor VIII and factor IX. 2020. Accessed 30 November, 2020. https://www.cdc.gov/ ncbddd/hemophilia/communitycounts/ data-reports/2020-9/table-2-factor.html

2. Srivastava A, Santagostino E, Dougall A, et al. WFH guidelines for the management of hemophilia, 3rd edition. Haemophilia. 2020;26(S6):1-158.
3. Ohmori T. Advances in gene therapy for hemophilia: basis, current status, and future perspectives. Int J Hematol. 2020;111(1):31-41.

4. Perrin GQ, Herzog RW, Markusic DM. Update on clinical gene therapy for hemophilia. Blood. 2019;133(5):407-14.

5. Quinn C, Young C, Thomas J, Trusheim M, Group MNFW. Estimating the clinical pipeline of cell and gene therapies and their potential economic impact on the US healthcare system. Value Health. 2019;22(6):621-26.

6. Pearson SD, Ollendorf DA, Chapman RH. New cost-effectiveness methods to determine value-based prices for potential cures: what are the options? Value Health. 2019;22(6):656-60.

7. Cook K, Forbes SP, Adamski K, Ma JJ, Chawla A, Garrison LP, Jr. Assessing the potential cost-effectiveness of a gene therapy for the treatment of hemophilia A. J Med Econ. 2020;23(5):501-12.

8. Machin N, Ragni MV, Smith KJ. Gene therapy in hemophilia A: a costeffectiveness analysis. Blood Adv. 2018;2(14):1792-98.

9. Rind D, Walton S, Agboola F, et al. Valoctocogene roxaparvovec and emicizumab for hemophilia A: effectiveness and value. Final report. Institute for Clinical and Economic Review. November 2020. Accessed December 4, 2021. https://icer.org/wp-content/ uploads/2020/10/ICER Hemophilia-A Final-Report_112020.pdf

10. Croteau SE, Cheng D, Cohen AJ, et al. Regional variation and cost implications of prescribed extended half-life factor concentrates among U.S. Haemophilia Treat. Centres for patients with moderate and severe haemophilia. Haemophilia. 2019;25(4):668-75.

11. Zhou ZY, Koerper MA, Johnson KA, et al. Burden of illness: direct and indirect costs among persons with hemophilia A in the United States. J Med Econ. 2015;18(6):457-65. 
12. Armstrong EP, Malone DC, Krishnan S, Wessler MJ. Costs and utilization of hemophilia A and B patients with and without inhibitors. J Med Econ. 2014;17(11):798-802.

13. Rocha P, Carvalho M, Lopes M, Araujo F. Costs and utilization of treatment in patients with hemophilia. BMC Health Serv Res. 2015;15:484.

14. Shrestha A, Eldar-Lissai A, Hou N, Lakdawalla DN, Batt K. Real-world resource use and costs of haemophilia A-related bleeding. Haemophilia. 2017;23(4):e267-75.

15. Patel A, Yang E, Raimundo K, Konkle B. Healthcare resource use and costs associated with arthropathy in hemophilia A. J Manag Care Spec Pharm. 2018;24(suppl 10-a):S33; abstract D8. Accessed December 4, 2021. https://www.jmcp.org/ doi/pdf/10.18553/jmcp.2018.24.10-a.s1

16. Chhabra A, Fogarty PF, Tortella BJ, et al. Real-world analysis of dispensed international units of coagulation factor VIII and resultant expenditures for hemophilia A patients: a comparison between standard half-life and extended half-life products. Manag Care. 2018;27(10):39-50.
17. Yang K, Buckley B, Sharma A, et al. Characterization of medical healthcare utilization among prophylaxis hemophilia A patients using a commercially-insured and Medicare advantage population claims database [P134]. Abstract presented at: 11th Annual Congress of the European Association for Haemophilia and Allied Disorders. February 7-9, 2018; Madrid, Spain.

18. Bowen K, Gleason P. Incremental cost of switching to extended half-life coagulation factor products to treat hemophilia among 15 million commercially insured members. J Manag Care Spec Pharm. 2018;24(suppl 4-a):S33; abstract D4. Accessed December 4, 2021. https://www.jmcp.org/doi/pdf/10.18553/ jmcp.2018.24.4-a.s1

19. Spurden D, Chhabra A, Tortella B, et al. Real world data analysis of US claims database on coagulation factor VIII expenditures in patients with hemophilia A: standard half-life versus extended half-life products. Value Health. 2018;21:S262.

20. Li NN, Glazebrook D, Su J. Real-world healthcare resource utilization of rFVIIIfc versus rFVIII in hemophilia A. Abstract presented at: Thrombosis and Hemostasis Societies of North America Summit. March 8-10, 2018; San Diego, CA.
21. Lyons J, Desai V, Xu Y, et al. Development and validation of an algorithm for identifying patients with hemophilia $\mathrm{A}$ in an administrative claims database. Value Health. 2018;21(9):1098-103.

22. Croteau SE, Cook K, Sheikh L, et al. Health care resource utilization and costs among adult patients with hemophilia A on factor VIII prophylaxis: an administrative claims analysis. J Manag Care Spec Pharm. 2021;27(3):316-26. doi:10.18553/ jmcp.2021.27.3.316

23. US Bureau of Labor Statistics. Consumer price index - all urban consumers, US medical care 2020. Accessed March 15, 2020. https://www. bls.gov/cpi/data.htm

24. Hemlibra. Prescribing information. Genentech; 2018. Accessed December 4, 2021. https://www.gene.com/download/ pdf/hemlibra_prescribing.pdf

25. Pasi KJ, Rangarajan S, Mitchell N, et al. Multiyear follow-up of AAV5-hFVIII-SQ gene therapy for hemophilia A. N Engl J Med. 2020;382(1):29-40.

26. Clinicaltrials.gov website. Study to evaluate the efficacy and safety of PF-07055480 in moderately severe to severe hemophilia A adults (AFFINE). Published April 30, 2020. Updated February 10, 2021. Accessed February 25, 2021. https://clinicaltrials.gov/ct2/show/ NCT04370054 